\title{
Vitamina D, CALcio TOTAl Y FósForo Digestible EN LA Nutrición PORCINA: RECOMENDACIONES NutricionAlES
}

\author{
Vitamin D, Total Calcium and Digestible Phosphorus in Swine \\ NUTRITION: NUTRITIONAL RECOMMENDATIONS
}

Jimmy Rolando Quisirumbay Gaibor* [e

Facultad de Medicina Veterinaria y Zootecnia, Universidad Central del Ecuador, Av. Universitaria, Quito, Ecuador

*Autor para correspondencia: jrquisirumbay@uce.edu.ec

Manuscrito recibido el 27 de noviembre de 2018. Aceptado, tras revisión, el 14 de enero de 2019. Publicado el 1 de marzo de 2019.

\section{Resumen}

La vitamina $\mathrm{D}$, calcio y fósforo son nutrientes esenciales necesarios para una mineralización ósea, inmuno-modulación y reproducción eficiente. La vitamina D se sintetiza en el organismo tras la exposición a la luz solar, además puede ser aportado a través de la dieta en sus formas D2 y D3. En los últimos años se ha utilizado la forma 25-OH-D3 en la alimentación animal con mejores resultados que la forma D3 debido a su mayor biodisponibilidad. Su deficiencia produce alteraciones en el desarrollo del sistema óseo conocido como raquitismo y osteomalacia en cerdos jóvenes y adultos, respectivamente. Existe un gran avance en el conocimiento de los requerimientos nutricionales de vitamina D, calcio y fósforo para la especie porcina, razón por la cual se han publicado varios artículos de investigación, revisión y guías nutricionales. Dentro de estas últimas destacan NRC, NSNG, Tablas Brasileñas, FEDNA y otras desarrolladas por la industria proveedora de aditivos y genética porcina de reconocido prestigio mundial. Los valores sugeridos varían entre las diferentes fuentes de información relacionadas en gran parte a las condiciones en las cuales fueron desarrolladas (experimentales, comerciales o tipo campo). Sin embargo, es el nutricionista quien debe estar en la capacidad de adaptar estos valores a sus condiciones y objetivos de producción. El propósito de este manuscrito es presentar de manera resumida las recomendaciones nutricionales de vitamina $\mathrm{D}$, calcio y fósforo proporcionando un contenido valioso para el nutricionista dedicado a la alimentación y formulación de dietas para cerdos.

Palabras clave: Alimentación, micronutrientes, vitaminas, minerales, cerdos.

\begin{abstract}
Vitamin D, calcium and phosphorus are essential nutrients necessary for efficient bone mineralization, immunomodulation and reproduction. Vitamin D is synthesized in the body after exposure to sunlight, and it can also be provided through the diet in its D2 and D3 forms. In recent years, the 25-OH-D3 form has been used in animal feed with better results than the D3 form due to its greater bioavailability. Its deficiency produces alterations in the development of the bone system known as rickets and osteomalacia in young and adult pigs, respectively. There is great
\end{abstract}


advance in the knowledge of the nutritional requirements of vitamin $\mathrm{D}$, calcium and phosphorus for the swine species, causing that research articles, reviews and nutritional guides have been published. Among the latter are NRC, NSNG, Brazilian Tables, FEDNA and others developed by the supplier industry of additives and genetics of swine of recognized world prestige. The suggested vales vary among the different sources of information related largely to the conditions in which they were developed (experimental, commercial or field type). However, it is the nutritionist who must be able to adapt these values to their production conditions and objectives. The purpose of this manuscript is to present in a summarized way the nutritional recommendations of vitamin $\mathrm{D}$, calcium and phosphorus providing a valuable content for the nutritionist dedicated to the feeding and formulation of diets for pigs.

Keywords: Food, micronutrients, vitamins, minerals, pigs.

Forma sugerida de citar: Quisirumbay Gaibor, J. R. (2019). Vitamina D, Calcio Total y Fósforo Digestible en la Nutrición Porcina: Recomendaciones Nutricionales. La Granja: Revista de Ciencias de la Vida. Vol. 29(1):6-16. http://doi.org/10.17163/lgr.n29.2019.01.

ID Orcid:

Jimmy Rolando Quisirumbay Gaibor: https:/ /orcid.org/0000-0003-1612-8503 


\section{Introducción}

La vitamina $D$ es una vitamina liposoluble que participa en la mineralización ósea (Endo y col., 2003) y está últimamente relacionada a procesos de inmuno-modulación (Bikle, 2008; Yuk y col., 2009; Baeke y col., 2010a; Baeke y col., 2010b; Sun, 2010; Zhao y col., 2014; Tian y col., 2016) y antioxidantes (Lahucky y col., 2007). Esta vitamina se acumula en el tejido adiposo de ratas, cerdos y humanos (Brouwer y col., 1998; Jakobsen y col., 2007; Didriksen y col., 2015), y es una molécula esteroidea que podría considerarse como una hormona en lugar de una vitamina debido a su mecanismo de acción.

La vitamina $\mathrm{D}$ se sintetiza en la piel, específicamente en las células epiteliales a partir del 7-deshidrocolesterol con intervención de la luz ultravioleta (Larson-Meyer y col., 2017). La activación de esta vitamina requiere de dos hidroxilaciones a nivel hepático y renal, en las posiciones C-25 y C-1, respectivamente, para formar un compuesto activo 1-25-( $\mathrm{OH})_{2}$-vitamina D (Hollis, 2005). La regulación de la activación a nivel renal se controla por la acción de la paratohormona (PTH) (Crenshaw, Rortvedt y Hassen, 2011; Dittmer y Thompson, 2011). El descenso en el nivel de calcio sérico estimula la secreción de PTH sintetizándose la vitamina D activa y provocando un aumento en el nivel de calcio plasmático (Cunningham, 2014). Este efecto se logra puesto que la vitamina $\mathrm{D}$ incrementa la $\mathrm{ab}-$ sorción de Ca y P a nivel intestinal, promueve una mayor resorción ósea y reduce la excreción renal de calcio y fosfato (Guyton y Hall, 2011). Adicionalmente la vitamina D puede ser ingerida a través de la dieta en su forma D2 o D3; los aceites de pescado naturalmente contienen grandes cantidades de la forma D3, mientras que la forma D2 está presente en ciertas plantas en forma de ergosterol, siendo transformado a la forma D2 por acción de la luz ultravioleta (DeLuca, 2004).

La producción porcina confinada limita la exposición a los rayos solares, causando un mayor riesgo de sufrir deficiencia de vitamina $\mathrm{D}$ aun cuando se esté suplementando a través de la dieta (Alexander y col., 2017), esto aunado al rápido crecimiento del cerdo y destetes tempranos. La deficiencia de vitamina $\mathrm{D}$ produce raquitismo, retraso en el desarrollo esquelético (Holick, 2006) y muscular en cerdos en crecimiento, mientras que en cerdos adultos la deficiencia produce osteomalacia (Pepper y col., 1978; Horst y Littledike, 1982; Fox y col., 1985; Thompson y Robinson, 1989; Dittmer y Thompson, 2011; NRC, 2012). Desde hace varios años se ha incorporado al mercado de la formulación de dietas una nueva forma de suplementación de vitamina $\mathrm{D}$, la 25OHD3 la cual al ser usada en cerdas mejora el estatus corporal de vitamina $\mathrm{D}$ al compararlo a la forma D3 (Fritts y Waldroup, 2003; Lauridsen y col., 2010; Coffey y col., 2012; Lauridsen, 2014), y está asociado a una mejor biodisponibilidad (Bar y col., 1980).

En cerdos de engorde, el desempeño productivo y la calidad de carne se ven mejorados cuando la fuente de vitamina D es el 25OHD3, relacionado en gran parte a su mejor biodisponibilidad (Jakobsen y col., 2007; Burild y col., 2016; Duffy y col., 2018). Sin embargo, dosis excesivamente altas pueden disminuir el consumo de alimento (Flohr y col., 2014b), y la suplementación dietaria de vitamina D3 en cerdas durante la gestación tiene efectos a largo plazo en el crecimiento, integridad y mineralización ósea de sus lechones. Algunas de estas alteraciones pueden estar presentes desde el nacimiento y otras pueden tardar más tiempo (3-8 semanas de edad) en manifestarse (Amundson y col., 2016). La suplementación de altas concentraciones de vitamina D3 en cerdas incrementa el nivel de 25(OH)D3 en el suero de cerdas y lechones, así como la concentración de vitamina D3 en leche y la concentración tisular del neonato (Flohr y col., 2014a). Por todo lo anterior es importante conocer los niveles correctos de suplementación de vitamina $\mathrm{D}$, calcio y fósforo en la alimentación porcina para mantener un estado de salud adecuada y garantizar un máximo rendimiento productivo.

En la actualidad existen diversas fuentes de información donde se pueden encontrar los requerimientos nutricionales para la especie porcina, unas de carácter académicoexperimental y otras creadas por la industria con el fin de alcanzar el máximo potencial productivo. En el primer grupo se encuentra la guía nutricional de la National Research Council (NRC, 2012), las Tablas brasileñas para aves y cerdos (Rostagno y col., 2017), la Guía Nacional de Nutrición de los Estados Unidos (NSNG) (U.S. Pork Center of Excellence, 2010), y las recomendaciones de la Fundación Española para el Desarrollo de la $\mathrm{Nu}$ trición Animal (FEDNA) (FEDNA, 2013). En el segundo grupo destaca la Guía de suplementación vitamínica 2016 de DSM para la nutrición animal (DSM, 2016) y el Manual de Especificación de Nutrientes de PIC (PIC, 2016). El objetivo de este manuscrito es presentar de manera resumida las recomendaciones nutricionales de vitamina $\mathrm{D}$, calcio y fósforo realizada por diferentes fuentes de reconocido prestigio mundial, proporcionando un contenido valioso para el nutricionista dedicado a la alimentación y formulación de dietas para cerdos en sus diferentes estados fisiológicos.

\section{Fuentes de información}

En la Tabla 1 se presentan las principales guías nutricionales utilizadas para la elaboración de este manuscrito y sus características. 
Tabla 1. Guías nutricionales

\begin{tabular}{|c|c|c|c|}
\hline Guía nutricional & Características & País de origen & $\begin{array}{l}\text { Año de } \\
\text { publicación }\end{array}$ \\
\hline $\begin{array}{l}\text { National Research } \\
\text { Council (NRC) }\end{array}$ & $\begin{array}{l}\text { Requerimientos nutricionales } \\
\text { mínimos. Desarrollado por } \\
\text { Universidades bajo condiciones } \\
\text { experimentales. }\end{array}$ & $\begin{array}{l}\text { Estados Unidos } \\
\text { de América }\end{array}$ & 2012 \\
\hline Tablas Brasileñas & $\begin{array}{l}\text { Requerimientos para una } \\
\text { producción más económica de } \\
\text { cerdos. Desarrollado por } \\
\text { Universidades del Brasil. }\end{array}$ & Brasil & 2017 \\
\hline $\begin{array}{l}\text { Guía Nacional de } \\
\text { Nutrición de los Estados } \\
\text { Unidos (NSNG) }\end{array}$ & $\begin{array}{l}\text { Proporciona márgenes de } \\
\text { seguridad para un rendimiento } \\
\text { productivo estándar. }\end{array}$ & $\begin{array}{l}\text { Estados Unidos } \\
\text { de América }\end{array}$ & 2010 \\
\hline $\begin{array}{l}\text { Fundación Española } \\
\text { para el Desarrollo de la } \\
\text { Nutrición Animal (FEDNA) }\end{array}$ & $\begin{array}{l}\text { Recomendaciones prácticas en } \\
\text { condiciones de campo para } \\
\text { alcanzar niveles de producción } \\
\text { estándar. }\end{array}$ & España & 2013 \\
\hline DSM (Comercial 1)* & $\begin{array}{l}\text { Recomendaciones para } \\
\text { alcanzar un máximo potencial } \\
\text { productivo en los cerdos. A } \\
\text { partir de investigaciones a nivel } \\
\text { universitario e industrial }\end{array}$ & Suiza & 2016 \\
\hline PIC (Comercial 2)* & $\begin{array}{l}\text { Recomendaciones para } \\
\text { alcanzar un máximo potencial } \\
\text { productivo en los cerdos. }\end{array}$ & $\begin{array}{l}\text { Estados Unidos } \\
\text { de América }\end{array}$ & 2016 \\
\hline
\end{tabular}

* Durante el desarrollo de este manuscrito se utilizarán los nombres de Comercial 1 y 2 para hacer referencia a las guías nutricionales de las empresas DSM y PIC, respectivamente.

\section{Requerimientos de vitamina $D$, calcio total (Ca total) y fósforo di- gestible ( $P$ dig.) para cerdos de engorde}

Los requerimientos de vitamina $\mathrm{D}$ se expresan en Unidades Internacionales (UI) por kilogramo de alimento, mientras que Ca total y $\mathrm{P}$ dig. se expresan en términos de composición porcentual (\%) (Tablas 2 y 3). No se incluyó el valor de fósforo total pues este no se utilizó en varias de las guías utilizadas. En la Figura 1 se grafican líneas de tendencia de las necesidades de vitamina D3 (eje vertical) considerando el peso del cerdo (eje horizontal).

Las necesidades de vitamina D según el NRC, (2012) son las más bajas de entre todas las guías nutricionales $150-220 \mathrm{UI} / \mathrm{Kg}$, pues incluyen la cantidad mínima necesaria para evitar el aparecimiento de signos clínicos relacionados a problemas deficitarios. Esto se opone con los niveles sugeridos por Comercial 2, 2233-3883 UI/Kg que es el más alto y corresponde a aproximadamente 14 a 17 veces por encima de lo reportado por el NRC, buscan- do alcanzar el mayor desempeño productivo del cerdo, proporcionando la cantidad necesaria para cubrir pérdidas por estrés en los animales, variaciones en la temperatura ambiental, presencia de enfermedades infecciosas, parasitosis entre otras situaciones que puedan mermar el rendimiento óptimo del animal. La guía U.S. Pork Center of Excellence, (2010) sugiere un nivel 3 veces superior al NRC encontrándose su rango entre $330-660 \mathrm{UI} / \mathrm{Kg}$ de alimento. Las Tablas brasileñas (2017), FEDNA (2013) y Comercial 1 (2016) presentan valores más cercanos entre sí, con un rango entre $1000-2700 U I / K g$, pero superiores a NRC, (2012) e inferiores a los reportados por Comercial 2. En todos los casos el aporte siempre es mayor en las fases iniciales de la vida del cerdo (etapa de pre y post-destete), pues el consumo de alimento es inferior al estar más concentrado el aporte de esta vitamina, logrando así satisfacer el nivel requerido. Mientras que, en las etapas de crecimiento y finalización, 30-60 Kg y $60-120 \mathrm{Kg}$ (o hasta la salida al mercado), respectivamente, disminuye el aporte de vitamina $\mathrm{D}$ por $\mathrm{Kg}$ de alimento, pero se incrementa el consumo de alimento por parte del animal, excepto en el caso de NSNG en el que aumenta el nivel de vitamina D 
Tabla 2. Recomendaciones nutricionales vitamina D3, Ca total, P dig. y relación Ca total-P dig. para NRC, Tablas Brasileñas y NSNG.

\begin{tabular}{cccccc}
\hline Guía nutricional & $\begin{array}{l}\text { Rango Peso } \\
\text { Vivo } \mathbf{( K g})\end{array}$ & $\begin{array}{l}\text { Vitamina D3 } \\
\text { (UI/Kg) }\end{array}$ & $\begin{array}{l}\text { Ca total } \\
(\boldsymbol{\%})\end{array}$ & $\begin{array}{l}\text { P digestible } \\
(\boldsymbol{\%})\end{array}$ & $\begin{array}{l}\text { Relación Ca } \\
\text { total-P dig. }\end{array}$ \\
\hline & $5-7$ & 220 & 0,85 & 0,7 & 1,21 \\
& $7-11$ & 220 & 0,8 & 0,65 & 1,23 \\
NRC (2012) & $11-25$ & 200 & 0,7 & 0,6 & 1,17 \\
& $25-50$ & 150 & 0,66 & 0,56 & 1,18 \\
& $50-75$ & 150 & 0,59 & 0,52 & 1,13 \\
& $75-100$ & 150 & 0,52 & 0,47 & 1,11 \\
& $100-135$ & 150 & 0,46 & 0,43 & 1,07 \\
Tablas Brasileñas (2017) & $3,5-5,5$ & 2707 & 0,888 & 0,5 & 1,78 \\
& $5,5-9$ & 2707 & 1,068 & 0,51 & 2,09 \\
& $9-15$ & 2405 & 0,973 & 0,47 & 2,09 \\
& $15-30$ & 1969 & 0,794 & 0,38 & 2,09 \\
& $30-50$ & 1599 & 0,655 & 0,31 & 2,09 \\
& $50-70$ & 1324 & 0,524 & 0,25 & 2,06 \\
& $70-100$ & 1101 & 0,454 & 0,22 & 2,06 \\
& $100-125$ & 924 & 0,406 & 0,197 & 2,06 \\
& $4-5$ & 660 & 0,9 & 0,57 & 1,58 \\
& $5-7$ & 660 & 0,85 & 0,53 & 1,6 \\
& $7-11$ & 660 & 0,85 & 0,4 & 2,13 \\
& $11-20$ & 660 & 0,75 & 0,33 & 2,27 \\
& $20-41$ & 550 & 0,71 & 0,29 & 2,45 \\
& $41-61$ & 550 & $0,61-0,65$ & $0,24-0,25$ & 2,57 \\
& $61-81$ & 440 & $0,56-0,61$ & $0,2-0,22$ & 2,79 \\
& $81-102$ & 440 & $0,52-0,57$ & $0,17-0,19$ & 3,03 \\
& $102-122$ & 330 & $0,49-0,53$ & $0,14-0,15$ & 3,52 \\
& $102-122^{*}$ & 550 & $0,51-0,55$ & $0,19-0,20$ & 2,72 \\
& $122-143^{*}$ & 550 & $0,48-0,52$ & $0,15-0,17$ & 3,13 \\
\hline
\end{tabular}

* Inclusión alimenticia de ractopamina.

en la etapa de acabado o finalización cuando se incluye ractopamina con la finalidad de tener un mayor desempeño magro en el cerdo.

En un estudio realizado en lechones con un peso inicial de $6,6 \mathrm{Kg}$ (28 días de edad) y una duración de 14 días se encontró que no existió diferencia significativa en el rendimiento productivo (consumo de alimento, ganancia diaria de peso, conversión alimentaria) ni en la mineralización ósea cuando la vitamina D3 se suplementó a niveles de 1378 (control) o $13780 \mathrm{UI} / \mathrm{Kg}$ de alimento. Sin embargo, cuando la suplementación se realizó a un nivel de $44100 \mathrm{UI} / \mathrm{Kg}$ (200 veces superior al NRC) el consumo de alimento disminuyó significativamente (Flohr y col., 2014b). Estos resultados coinciden con los reportados en otro estudio realizado por el mismo autor, en el cual la suplementación de vitamina D3 a niveles de 1800 o $18000 \mathrm{UI} / \mathrm{Kg}$ durante 35 días posteriores al destete (día
21 edad) no generó diferencias significativas en las variables productivas (Flohr y col., 2014a). Adicionalmente, la suplementación de vitamina D3 a un nivel de $3500 \mathrm{UI} / \mathrm{Kg}$ en lechones destetados 6-7 $\mathrm{kg}$ de peso (18 días de edad) provoca un impacto inmuno-modulador al aumentar significativamente el número de leucocitos en sangre y su capacidad fagocítica (Konowalchuk y col., 2013). La suplementación de vitamina D3 a niveles supranutricionales de $40000 \mathrm{u} 80000 \mathrm{UI}$ por $\mathrm{Kg}$ de alimento por al menos 44 días previo al sacrificio mejora el color de la carne e incrementa el $\mathrm{pH}$ sin afectar las concentraciones de calcio muscular (Wilborn y col., 2004). Por su parte, Wiegand y col., (2002) encontraron que la suplementación de niveles elevados de vitamina D3 (250000-500000 UI/día) en cerdos en finalización 3 días previos al sacrificio resulta en altas concentraciones plasmáticas de calcio sin mejorar la calidad de la carne. 
Tabla 3. Recomendaciones nutricionales de la vitamina D3, Ca total, P dig. y relación Ca total-P dig. para FEDNA, Comercial 1 y 2.

\begin{tabular}{|c|c|c|c|c|c|}
\hline $\begin{array}{l}\text { Guía } \\
\text { nutricional }\end{array}$ & $\begin{array}{l}\text { Rango Peso } \\
\text { Vivo (Kg) }\end{array}$ & $\begin{array}{l}\text { Vitamina D3 } \\
\text { (UI/Kg) }\end{array}$ & $\begin{array}{l}\text { Ca total } \\
(\%)\end{array}$ & $\begin{array}{l}\text { P digestible } \\
(\%)\end{array}$ & $\begin{array}{l}\text { Relación } \\
\text { Ca total-P dig. }\end{array}$ \\
\hline \multirow{6}{*}{$\begin{array}{l}\text { FEDNA } \\
(2013)\end{array}$} & $5-7$ & 1800 & $0,65-0,75$ & 0,41 & 1,71 \\
\hline & $7-12$ & 1800 & $0,70-0,80$ & 0,38 & 1,97 \\
\hline & $12-20$ & 1800 & $0,73-0,81$ & 0,33 & 2,33 \\
\hline & $20-60$ & 1250 & $0,67-0,80$ & 0,28 & 2,63 \\
\hline & $60-100$ & 1100 & $0,65-0,80$ & 0,25 & 2,9 \\
\hline & $>100$ & 1100 & $0,59-0,80$ & 0,23 & 3,02 \\
\hline \multirow{4}{*}{$\begin{array}{l}\text { Comercial } 1 \\
(2016)\end{array}$} & $<5$ & $1800-2000$ & - & - & - \\
\hline & $5-30$ & $1800-2000$ & - & - & - \\
\hline & $30-70$ & $1500-2000$ & - & - & - \\
\hline & 70 - mercado & $1000-1500$ & - & - & - \\
\hline \multirow{11}{*}{$\begin{array}{l}\text { Comercial } 2 \\
(2016)\end{array}$} & $3,5-5,5$ & 3883 & 0,85 & 0,57 & 1,49 \\
\hline & $5,5-7,5$ & 3883 & 0,85 & 0,57 & 1,49 \\
\hline & $7,5-11,5$ & 3883 & 0,79 & 0,44 & 1,8 \\
\hline & $11,5-23$ & 3883 & 0,71 & 0,39 & 1,82 \\
\hline & $23-40$ & 2673 & $0,70-0,71$ & 0,33 & 2,14 \\
\hline & $40-60$ & 2673 & $0,64-0,65$ & 0,3 & 2,15 \\
\hline & $60-80$ & 2673 & $0,58-0,6$ & $0,27-0,28$ & 2,15 \\
\hline & $80-105$ & 2233 & $0,53-0,55$ & $0,25-0,26$ & 2,12 \\
\hline & 105 - mercado & 2233 & $0,48-0,5$ & 0,24 & 2,04 \\
\hline & $\begin{array}{l}105-\text { mercado } \\
(<21 \text { días })^{*}\end{array}$ & 2233 & $0,63-0,64$ & $0,29-0,30$ & 2,15 \\
\hline & $\begin{array}{l}105-\text { mercado } \\
(>21 \text { días })^{*}\end{array}$ & 2233 & $0,60-0,63$ & $0,28-0,29$ & 2,16 \\
\hline
\end{tabular}

* Inclusión alimenticia de ractopamina.

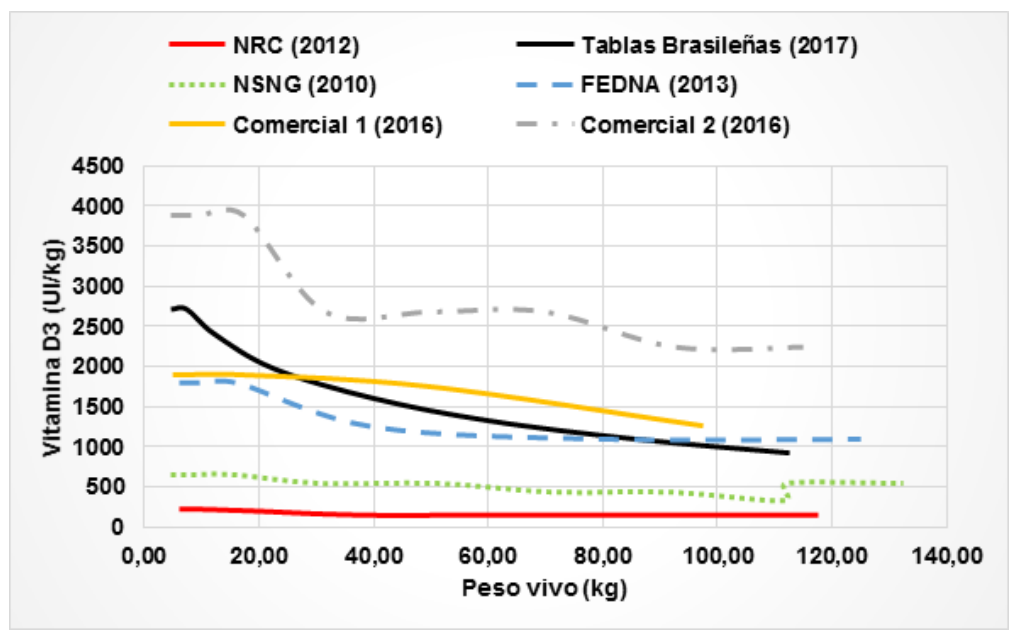

Figura 1. Requerimiento de vitamina D3 durante el crecimiento del cerdo. 


\section{Relación Ca total (\%) - P digesti- 5 Recomendaciones nutricionales ble $(\%)$ en cerdos de engorde \\ La relación Ca total-P dig. (Tablas 2 y 3) resulta de dividir de Vitamina D3, calcio total y fós- foro digestible para cerdos en eta- pa reproductiva} el valor de la columna Ca total para el valor de la columna $\mathrm{P}$ dig. y se consideró como una variable referencial para poder comparar entre las diferentes fuentes de información; además se ha graficado la tendencia de la relación considerando los requerimientos a medida que el cerdo va creciendo (Figura 2). La primera de ellas NRC con una recta sostenida a lo largo del crecimiento del cerdo con valores superiores a $1(1,07-1,21)$, sin importantes variaciones hasta llegar al peso de faena. Las Tablas brasileñas y Comercial 2 presentan también una recta sostenida pero sus valores están muy próximos a 2; 1,78-2,09 y $1,49-2,16$ respectivamente. Por su parte U.S. Pork Center of Excellence, (2010) y FEDNA, (2013) presentan una tendencia al aumento a medida que el cerdo crece, llegando a alcanzar una relación de 3 o más. Valores más altos de la relación Ca total $-\mathrm{P}$ digestible resultan de una gran amplitud entre los niveles de Ca sugerido y los requerimientos de $\mathrm{P}$, específicamente altos requerimientos de $\mathrm{Ca}$ y más bajos de P. No fue posible presentar la tendencia de Comercial 1 pues esta guía únicamente hace recomendaciones de vitaminas.
Las recomendaciones de vitamina $\mathrm{D}$ para cerdos en etapa reproductiva se muestran en la Tabla 4 y se grafican en la Figura 3 para mejor comprensión. Los valores más bajos son los sugeridos por el NRC en cerdas de reemplazo (nulíparas o futuras madres) y verracos (machos sexualmente activos) siendo de $200 \mathrm{UI} / \mathrm{Kg}$ para ambos casos, mientras que para el caso de cerdas los valores más bajos los presenta NSNG $660 \mathrm{UI} / \mathrm{Kg}$. Los niveles más altos en todas las categorias reproductivas están dado por Comercial 21985,1985 y 2000 UI/Kg para reemplazo, cerdas y verracos, respectivamente; resultando ser 10 veces superior a las recomendaciones del NRC y coincidiendo en ser el nivel más alto por parte de esta guía al igual que en cerdos de engorde. Seguido a los valores de Comercial 2 se encuentran los de Comercial 1, 1900, 1750 y $1750 \mathrm{UI} / \mathrm{Kg}$ de alimento. Valores intermedios en la comparación general y muy similares entre sí son los descritos por las tablas brasileñas y FEDNA con un rango entre $1200-1600 \mathrm{UI} / \mathrm{Kg}$; siendo 8 veces superior a las recomendaciones del NRC para reemplazo y verracos y el doble en la categoría de cerdas.

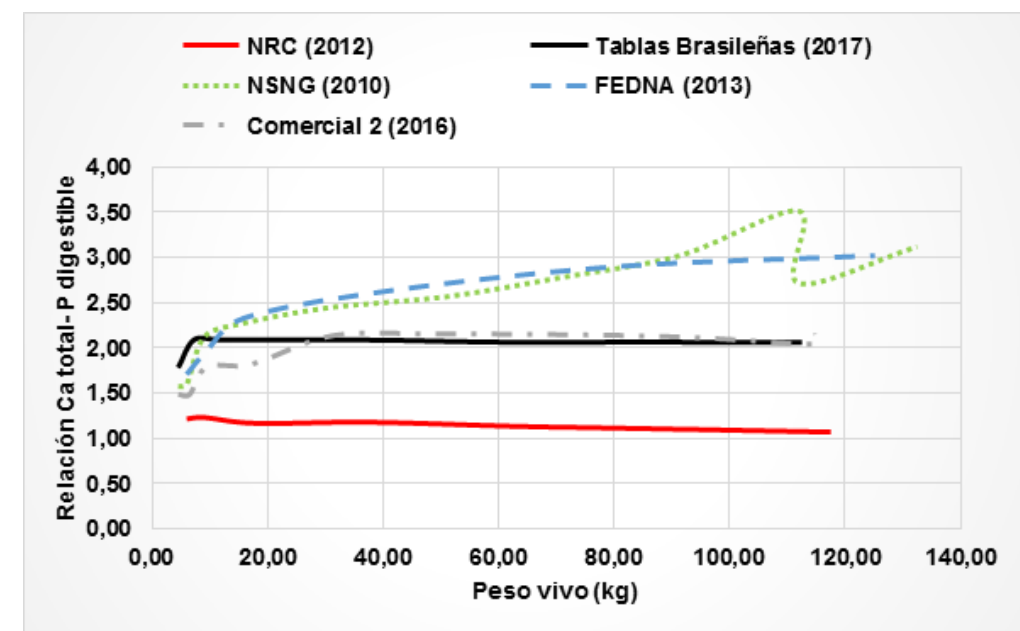

Figura 2. Relación Ca total - P digestible durante el crecimiento de cerdos. 
Tabla 4. Recomendaciones nutricionales de vitamina D3, Ca total y P dig. en cerdos en etapa reproductiva.

\begin{tabular}{cccccc}
\hline $\begin{array}{l}\text { Guía } \\
\text { nutricional }\end{array}$ & Etapa & $\begin{array}{l}\text { Vitamina D } \\
(\mathbf{U I} / \mathbf{K g})\end{array}$ & $\begin{array}{l}\text { Ca total } \\
(\boldsymbol{\%})\end{array}$ & $\begin{array}{l}\text { P digestible } \\
(\boldsymbol{\%})\end{array}$ & $\begin{array}{l}\text { Relación Ca } \\
\text { total-P dig. }\end{array}$ \\
\hline \multirow{2}{*}{ NRC } & Reemplazo & 200 & $0,49-0,61$ & $0,27-0,30$ & 1,93 \\
$(2012)$ & Cerdas & 800 & $0,43-0,83$ & $0,19-0,36$ & 2,29 \\
& Verracos & 200 & $0,57-0,64$ & $0,23-0,28$ & 2,37 \\
\hline Tablas & Reemplazo & 1560 & $0,705-0,847$ & $0,333-0,405$ & 2,1 \\
Brasileñas & Cerdas & 1560 & $0,705-0,847$ & $0,333-0,405$ & 2,1 \\
$(2017)$ & Verracos & 1560 & $0,705-0,847$ & $0,333-0,405$ & 2,1 \\
\hline \multirow{2}{*}{ NSNG } & Reemplazo & 660 & $0,65-0,81$ & $0,26-0,35$ & 2,39 \\
$(2010)$ & Cerdas & 660 & $0,85-0,90$ & $0,35-0,39$ & 2,36 \\
& Verracos & 660 & $0,68-0,86$ & $0,29-0,45$ & 2,08 \\
\hline \multirow{2}{*}{ FEDNA } & Reemplazo & 1600 & $0,85-1,05$ & $0,30-0,32$ & 3,06 \\
$(2013)$ & Cerdas & 1600 & $0,81-1,05$ & $0,29-0,32$ & 3,05 \\
& Verracos & 1200 & $0,85-1$ & 0,31 & 2,98 \\
\hline \multirow{2}{*}{ Comercial 1 } & Reemplazo & 1900 & - & - & - \\
$(2016)$ & Cerdas & 1750 & - & - & - \\
& Verracos & 1750 & - & - & - \\
\hline \multirow{2}{*}{ Comercial 2 } & Reemplazo & 1985 & 0,7 & 0,35 & 2 \\
$(2016)$ & Cerdas & 1985 & 0,85 & 0,44 & 1,93 \\
& Verracos & 2000 & 0,8 & 0,4 & 2 \\
\hline
\end{tabular}

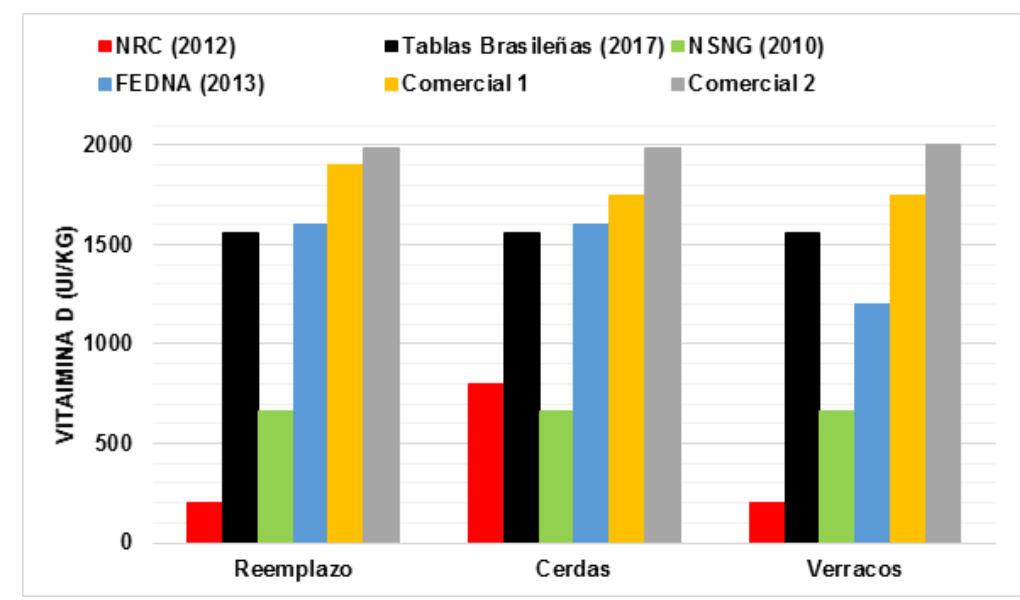

Figura 3. Requerimientos de vitamina D3 en cerdos en etapa reproductiva.

La suplementación de 25OHD3 (500 IU D3/ Kg de alimento $+50 \mathrm{~g} 25 \mathrm{OHD} 3 / \mathrm{Kg}$ de alimento) en cerdas de primer servicio antes y durante la gestación mejora el estatus de vitamina $\mathrm{D}$ en la madre y en los fetos, la tasa de concepción y el tamaño de la camada sin afectar la media del peso vivo de los lechones en comparación con las cerdas que únicamente recibieron vitamina D3 $(2500 \mathrm{UID} 3 / \mathrm{Kg}$ de alimento) (Coffey y col., 2012). En un estudio similar se encontró que el 25OHD3 suplementado en los mismos niveles del estudio anterior en cerdas gestantes impacta positivamente en el desarrollo muscular esquelético fe- tal así como también en la actividad mioblástica (Hines y col., 2013; Zhou y col., 2016). Lo anterior se debe a que la forma 25OHD3 tiene mayor biodisponibilidad en comparación con la forma D3 (Sitrin y col., 1982; Bischoff-Ferrari y col., 2012), sin embargo, independientemente de la forma de la vitamina $\mathrm{D}$ y de la dosis, la transferencia de esta vitamina a la progenie es escasa por vía transplacentaria (Lauridsen y col., 2010) no así a través de la leche materna (Flohr y col., 2016a; Flohr y col., 2016b). Adicionalmente, en un estudio realizado en cerdas en las cuales se les suplementó $250 H D 3$ a un nivel de $2000 \mathrm{UI} / \mathrm{Kg}$ durante la 
gestación y lactancia se mejoró el estatus de esta vitamina en las cerdas y la mineralización esquelética en los lechones (Witschi y col., 2011), a este mismo nivel pero en otro estudio se determinó que la suplementación de 25OHD3 a una concentración $2000 \mathrm{UI} / \mathrm{Kg}$ incrementó de manera significativa el peso al nacimiento y al destete, comparado con la forma D3 (Weber y col., 2014). Por su parte en verracos la suplementación de vitamina D3 a un nivel de $2000 \mathrm{UI} / \mathrm{Kg}$ mejora la calidad de los espermatozoides, relacionado a un incremento en la síntesis hormonal y a cambios en la composición del plasma seminal y a la expresión genética en los espermatozoides; estadísticamente no hubo diferencia entre la suplementación de 2000 UI con 4000 UI de vitamina D3 por $\mathrm{Kg}$ de alimento (Lin y col., 2017).

\section{Relación Calcio total - fósforo di- gestible en cerdos en etapa repro- ductiva}

Las relaciones calcio total-fósforo digestible se observan en la Figura 4. Los valores más altos son los calculados para FEDNA siendo de 3,06, 3,05 y 2,98 en reemplazo, cerdas y verracos, respectivamente. Las demás guías nutricionales (NRC, Tablas brasileñas, NSNG, Comercial 2) presentan valores similares que oscilan entre $1,93-2,39 ; 1,93-2,36$ y 2 2,37 para reemplazo, cerdas y verracos respectivamente. No se presentan los valores de Comercial 1 pues esta guía únicamente presenta recomendaciones de vitaminas.

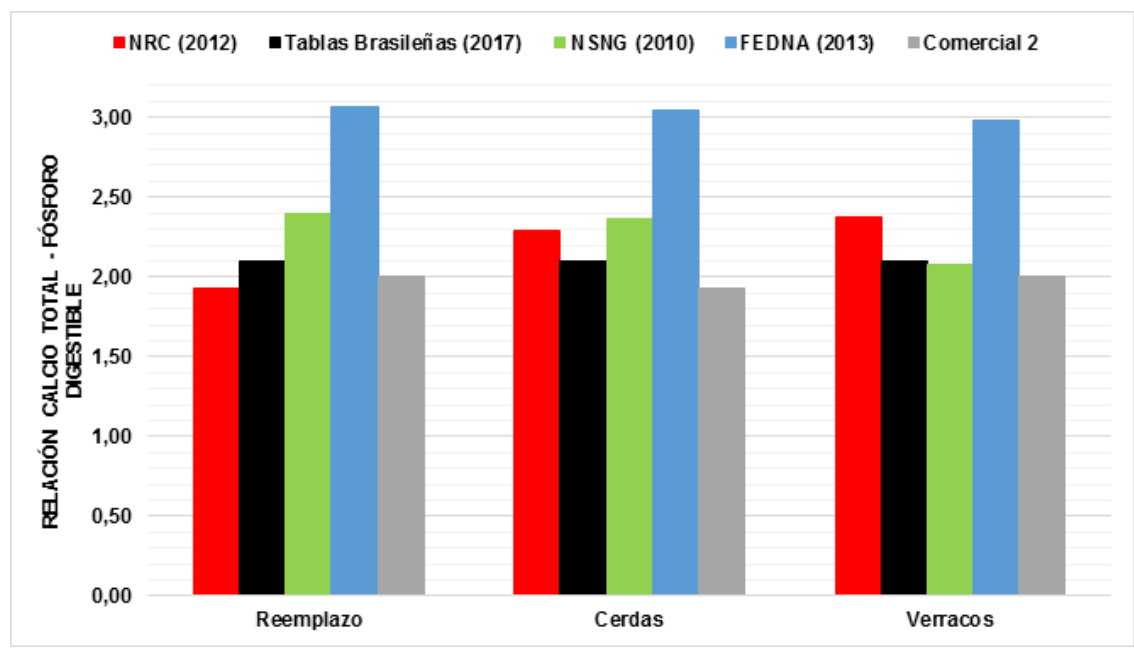

Figura 4. Relación Ca total-P digestible para cerdos en etapa reproductiva.

\section{Conclusión}

Varias son las fuentes de información (guías nutricionales) de las cuales se pueden obtener las recomendaciones nutricionales para cerdos en etapas productiva (engorde) y reproductiva. Amplia es la variación existente entre las sugerencias de vitamina $\mathrm{D}$, calcio total y fósforo digestible entre las distintas referencias, el nutricionista y/o especialista en producción porcina debe estar en la capacidad de adaptar los niveles de nutrientes sugeridos a las condiciones de producción en las cuales trabaja, buscando de esta manera alcanzar los objetivos de la granja.

\section{Referencias}

Alexander, B.M. y col. (2017). «Sunlight exposure increases vitamin D sufficiency in growing pigs fed a diet formulated to exceed requirements». En: Domestic Animal Endocrinology 59, 37 -43. Online:https:/ / bit. ly/2Ay4bfU.

Amundson, Laura A. y col. (2016). «Maternal dietary vitamin D carry-over alters offspring growth, skeletal mineralisation and tissue mRNA expressions of genes related to vitamin D, calcium and phosphorus homoeostasis in swine». En: British Journal of Nutrition 116.5, 774-787. Online:https:/ / bit.ly/2Dbq8Eb.

Baeke, Femke y col. (2010a). «Human T lymphocytes are direct targets of 1,25-dihydroxyvitamin D3 in the immune system». En: The Journal of Steroid Biochemistry and Molecular Biology 121.1. Proceedings of the 14th Vitamin D Workshop, 221-227. Online:https:/ / bit.ly / 2JlNbMG.

Baeke, Femke y col. (2010b). «Vitamin D: modulator of the immune system». En: Current Opinion in Pharmacology. 
Bar, Arie y col. (1980). «Absorption and Excretion of Cholecalciferol and of 25-Hydroxycholecalciferol and Metabolites in Birds». En: The Journal of Nutrition 110.10, 1930-1934. Online:https:/ / bit.ly/2zaLEoe.

Bikle, Daniel D (2008). «Vitamin D and the immune system: role in protection against bacterial infection». En: Current Opinion in Nephrology and Hypertension 17.4, 348-352. Online: https:/ / bit.ly/2D82Yi9.

Bischoff-Ferrari, H. A. y col. (2012). «Oral supplementation with $25(\mathrm{OH}) \mathrm{D} 3$ versus vitamin D3: effects on 25(OH)D levels, lower extremity function, blood pressure, and markers of innate immunity.» En: Journal of Bone and Mineral Research 27.1, 160-169. Online:https: / /bit.ly/2StwzXq.

Brouwer, D. A. Janneke y col. (1998). «Rat adipose tissue rapidly accumulates and slowly releases an orallyadministered high vitamin D dose.» En: British Journal of Nutrition 79.6, 527-532. Online:https: / / bit.ly / 2JnOw5U.

Burild, Anders y col. (2016). «Vitamin D3 and 25hydroxyvitamin D3 in pork and their relationship to vitamin D status in pigs». En: Journal of Nutritional Science 5.e3, 1-6. Online:https:/ / bit.ly/2OXbOWg.

Coffey, J. D. y col. (2012). «Feeding 25hydroxycholecalciferol improves gilt reproductive performance and fetal vitamin D status». En: Journal of Animal Science 90.11, 3783-3788. Online:https: / / bit.ly/2RjApkA.

Crenshaw, T. D., L. A. Rortvedt y Z. Hassen (2011). «Triennial Growth Symposium: a novel pathway for vitamin D-mediated phosphate homeostasis: implications for skeleton growth and mineralization.» En: Journal of animal science 89.7, 1957-1964. Online:https: / / bit.ly/2CPgeY0.

Cunningham J. G. \& Klein, B. G (2014). Fisiología Veterinaria. Barcelona: Elsevier. Online:https : / / bit.ly / 2zes5uW.

DSM, ed. (2016). Guía de Suplementación Vitamínica para la nutrición animal, Online:https:/ / bit.ly/2Oe1uUi.

DeLuca, Hector F (2004). «Overview of general physiologic features and functions of vitamin D». En: The American Journal of Clinical Nutrition 80.6, 1689S-1696S. Online:https://bit.ly/2OWyE04 .

Didriksen, Allan y col. (2015). «Vitamin D3 increases in abdominal subcutaneous fat tissue after supplementation with vitamin D3». En: European Journal of Endocrinology 172.3, 235 -241. Online:https: / / bit.ly / 2Dbc4L4.

Dittmer, K. E. y K. G. Thompson (2011). «Vitamin D Metabolism and Rickets in Domestic Animals: A Review». En: Veterinary Pathology 48.2. PMID: 20634407, 389-407. Online:https:/ / bit.ly/2Q7J7m5.

Duffy, Sarah K. y col. (2018). «The use of synthetic and natural vitamin D sources in pig diets to improve meat quality and vitamin D content». En: Meat Science 143, 60-68. Online:https://bit.ly/2RnpIOa.
Endo, Itsuro y col. (2003). «Deletion of Vitamin D Receptor Gene in Mice Results in Abnormal Skeletal Muscle Development with Deregulated Expression of Myoregulatory Transcription Factors». En: Endocrinology 144.12, 5138-5144. Online:https:/ / bit.ly/2yA96f5.

FEDNA (2013). Necesidades Nutricionales para Ganado Porcino: Normas FEDNA. Ed. por FEDNA. Segunda. Fundación Española para el Desarrollo de la Nutrición Animal. Online:https://bit.ly/2Px5HHq.

Flohr, J. R. y col. (2014a). «An evaluation of the effects of added vitamin D3 in maternal diets on sow and pig performance». En: Journal of Animal Science 92.2, 594-603. Online:https:/ / bit.ly/2Q36GMI.

Flohr, J. R. y col. (2014b). «Effects of supplemental vitamin D3 on serum 25-hydroxycholecalciferol and growth of preweaning and nursery pigs». En: Journal of animal science 92.1, 152-63. Online:https: / / bit.ly /2SqWhMh.

Flohr, J. R. y col. (2016a). «Evaluating the impact of maternal vitamin D supplementation: I. Sow performance, serum vitamin metabolites, and neonatal muscle characteristics». En: Journal of Animal Science 94.11, 4629-4642. Online:https:/ / bit.ly/2StiOIn.

- (2016b). «Evaluating the impact of maternal vitamin D supplementation on sow performance: II. Subsequent growth performance and carcass characteristics of growing pigs». En: Journal of Animal Science 94.11, 4643-4653. Online:https:// bit.ly/2COsw2D.

Fox, John y col. (1985). «Vitamin D-dependent rickets type I in pigs». En: Clinical Science. 0143-5221 69.5, 541-548. Online:https:/ / bit.ly/2ESPI2p.

Fritts, C. A. y P. W. Waldroup (2003). «Effect of Source and Level of Vitamin D on Live Performance and Bone Development in Growing Broilers». En: The Journal of Applied Poultry Research 12.1, 45-52. Online:https: / / bit.ly/2Q2W7ZX.

Guyton, A. C. y J. E Hall (2011). Fisiología Médica. Barcelona: Elsevier. Online:https:/ / bit.ly/2PYDs1o.

Hines, E. A. y col. (2013). «Improvement of maternal vitamin D status with 25-hydroxycholecalciferol positively impacts porcine fetal skeletal muscle development and myoblast activity». En: Journal of Animal Science 91.9, 4116-4122. Online:https: / / bit.ly / 2OdleHK.

Holick, M. F. (2006). «Resurrection of vitamin D deficiency and rickets». En: The Journal of clinical investigation 116.8, 2062-72. Online:https:/ / bit.ly/2CQGSQj.

Hollis, Bruce W. (2005). «Circulating 25-Hydroxyvitamin D Levels Indicative of Vitamin D Sufficiency: Implications for Establishing a New Effective Dietary Intake Recommendation for Vitamin D». En: The Journal of Nutrition 135.2, 317-322. Online:https: / / bit.ly / 2yD6mO8.

Horst, R.L. y E.T. Littledike (1982). «Comparison of plasma concentrations of vitamin D and its metabolites in young and aged domestic animals». En: Comparative 
Biochemistry and Physiology Part B: Comparative Biochemistry 73.3, 485-489. Online:https: / / bit.ly/2ESiWyB.

Jakobsen, Jette y col. (2007). «25-Hydroxyvitamin D3 affects vitamin D status similar to vitamin D3 in pigsbut the meat produced has a lower content of vitamin D». En: British Journal of Nutrition 98.5, 908-913. Online:https:/ / bit.ly/2Sr218N.

Konowalchuk, Jeffrey D. y col. (2013). «Modulation of weanling pig cellular immunity in response to diet supplementation with 25-hydroxyvitamin D3». En: Veterinary Immunology and Immunopathology 155.1, 57-66. Online:https://bit.ly/2qigsiD.

Lahucky, Rudolf y col. (2007). «Effects of dietary supplementation of vitamins D3 and E on quality characteristics of pigs and longissimus muscle antioxidative capacity». En: Meat Science 77.2, 264-268. Online:https: / / bit.ly/2RliSZc.

Larson-Meyer, D. Enette y col. (2017). «Sun exposure in pigs increases the vitamin $\mathrm{D}$ nutritional quality of pork». En: PLOS ONE 12.11, 1-12. Online:https://bit. ly/2ERq7qJ.

Lauridsen, C. (2014). «TRIENNIAL GROWTH SYMPOSIUM -Establishment of the 2012 vitamin D requirements in swine with focus on dietary forms and levels of vitamin D1». En: Journal of Animal Science 92.3, 910-916. Online:https://bit.ly/2Sr2Hel.

Lauridsen, C. y col. (2010). «Reproductive performance and bone status markers of gilts and lactating sows supplemented with two different forms of vitamin D1». En: Journal of Animal Science 88.1, 202-213. Online:https:/ / bit.ly/2qfhr30.

Lin, Yan y col. (2017). «Effects of the different levels of dietary vitamin D on boar performance and semen quality». En: Livestock Science 203, 63-68. Online:https: / / bit.ly/2OVHOd7.

NRC, National Research Council (2012). Nutrient Requirements of Swine: Eleventh Revised Edition. Ed. por National Research Council. Washington, DC: National Academies Press.

PIC (2016). Manual de especificación de nutrientes. Ed. por PIC. PIC. Online:https://bit.ly/2Rm6VTf.

Pepper, TA y col. (1978). «Rickets in growing pigs and response to treatment». En: Veterinary Record 103.1, 4-8. Online:https://bit.ly/2z8rjjg.

Rostagno, H. S. y col. (2017). Tablas brasileñas para aves y cerdos : composición de alimentos y requerimientos nutricionales. Ed. por Departamento de Zootecnia Universidad Federal de Viçosa. Cuarta. Brasil: Universidad Federal de Viçosa, Departamento de Zootecnia. Online:https:/ / bit.ly/2EO9xI8.

Sitrin, M. D. y col. (1982). "Comparison of vitamin D and 25-hydroxyvitamin D absorption in the rat». En:
American Journal of Physiology-Gastrointestinal and Liver Physiology 242.4. PMID: 6978078, G326-G332. Online:https:/ / bit.ly/2yEmHlm.

Sun, Jun (2010). "Vitamin D and mucosal immune function». En: Current opinion in gastroenterology 26.6, 591-595. Online:https://bit.ly/2Azgl8y.

Thompson, K. G. y B. M Robinson (1989). «An osteodystrophy apparently caused by vitamin D deficiency in growing pigs». En: New Zealand Veterinary Journal 37.4, 155-157. Online:https://bit.ly/2Azgl8y.

Tian, Gang y col. (2016). «Vitamin D3 supplementation alleviates rotavirus infection in pigs and IPEC-J2 cells via regulating the autophagy signaling pathway». En: The Journal of Steroid Biochemistry and Molecular Biology 163, 157-163. Online:https:/ / bit.ly/2Sqak4O.

U.S. Pork Center of Excellence (2010). National Swine Nutrition Guide. Ed. por U.S. Pork Center of Excellence. NSNG. Online:https://bit.ly/2OXX21d.

Weber, G. M. y col. (2014). «Triennial Growth Symposium- Effects of dietary 25-hydroxycholecalciferol and cholecalciferol on blood vitamin $\mathrm{D}$ and mineral status, bone turnover, milk composition, and reproductive performance of sows». En: Journal of Animal Science 92.3, 899-909. Online:https: / / bit.ly/2qj3kKk.

Wiegand, B. R. y col. (2002). «Short-term feeding of vitamin D3 improves color but does not change tenderness of pork-loin chops.» En: Journal of Animal Science 80.8, 2116-2121. Online:https://bit.ly/2Q2dLwT.

Wilborn, B. S. y col. (2004). "Improving pork quality by feeding supranutritional concentrations of vitamin D3.» En: Journal of Animal Science 82.1, 218-224. online:https:/ / bit.ly/2RpdHI5.

Witschi, A.-K. M. y col. (2011). «Effect of source and quantity of dietary vitamin $\mathrm{D}$ in maternal and creep diets on bone metabolism and growth in piglets». En: Journal of Animal Science 89.6, 1844-1852. Online:https: / / bit.ly /2qjapdO.

Yuk, J.M. y col. (2009). «Vitamin D3 induces autophagy in human monocytes/macrophages via cathelicidin.» En: Cell Host Microbe 6.3, 231-243. Online:https: / / bit. ly/2qiIIBQ.

Zhao, Ye y col. (2014). «Dietary vitamin D supplementation attenuates immune responses of pigs challenged with rotavirus potentially through the retinoic acidinducible gene I signalling pathway». En: British Journal of Nutrition 112.3, 381-389. Online:https: / / bit.ly / 2JnsDDD.

Zhou, Hui y col. (2016). «Improving maternal vitamin D status promotes prenatal and postnatal skeletal muscle development of pig offspring». En: Nutrition 32.10, 1144-1152. Online:https:/ / bit.ly/2Oed1TC. 